\title{
Metallization of Atomic Solid Hydrogen within the Extended Hubbard Model with Renormalized Wannier Wave Functions
}

\author{
A.P. KĄDZIELAWA* \\ Marian Smoluchowski Institute of Physics, Jagiellonian University, W.S. Reymonta 4, PL-30-059 Kraków, Poland
}

\begin{abstract}
We refer to our recent calculations Eur. Phys. J. B 86, 252 (2013) of metallization pressure of the threedimensional simple-cubic crystal of atomic hydrogen and study the effect on the crucial results concocting from approximating the $1 s$ Slater-type orbital function with a series of $p$ Gaussians. As a result, we find the critical metallization pressure $p_{\mathrm{C}}=102 \mathrm{GPa}$. The latter part is a discussion of the influence of zero-point motion on the stabilizing pressure. We show that in our model the estimate magnitude of zero-point motion carries a little effect on the critical metallization pressure at zero temperature.
\end{abstract}

DOI: 10.12693/APhysPolA.126.A-58

PACS: 71.30.+h, 71.27.+a, 71.10.Fd, 62.50.-p

\section{Motivation}

This year we are celebrating the 50th anniversary of the Hubbard model, a second-quantization language to describe strongly correlated systems provided independently by Hubbard [1], Gutzwiller [2,3] and Kanamori [4]. This description shed some light on many-body quantum systems, in particular on the localizationdelocalization transitions of fermions states in the solidstate [5-8], and optical-lattice [9] systems. This transition is called the Mott or Mott-Hubbard transition.

In the series of papers [10-12], we have conducted model calculations combining both the Mott [5] and the Hubbard [13] aspects of the phase transition, within the extended Hubbard model, with a simultaneous renormalization of the single-particle Wannier basis, connecting first- and second-quantization approach. In [12] we obtained, using proposed model, the critical metallization pressure $p_{\mathrm{C}}=97.7 \mathrm{GPa}$ required to stabilize the atomic-hydrogen-like crystal, while having both the Mott $\left(n_{\mathrm{C}}^{1 / 3} a_{\mathrm{B}} \approx 0.2\right)$ and the Hubbard $(U \approx W)$ criteria satisfied at the same time. Thus, those two criteria represent two sides of the same coin.

Ever since Ashcroft proposed an explanation for greater-than-expected magnetic field of Jovian planets [14] by applying the BCS theory to the metallic hydrogen, the pursuit of the metallization of this element began. Predicted by Wigner and Huntington in 1935 [15] the conducting phase of hydrogen is claimed to have various properties, including hypothesis of being superconducting up to the room temperature [14].

In this paper we briefly describe the model in Sect. 2 . Then in Sect. 3 we review the validity of approximations made in [12] and show that they were in fact sufficient (explicitly redoing all calculations and showing no

\footnotetext{
*e-mail: kadzielawa@th.if.uj.edu.pl
}

qualitative changes). We also show that both Mott and Hubbard criteria of localization-delocalization transition are satisfied. In Sect. 4 we estimate the magnitude of zero-point motion energy, omitted in our calculations to test the strength of our results, keeping in mind the possibility of quantum melting of hydrogen.

\section{Model}

We start with the extended Hubbard Hamiltonian describing a single-band hydrogen system [10-12]:

$$
\begin{aligned}
\mathcal{H} & =\epsilon_{a} \sum_{i} n_{i}+\sum_{i \neq j, \sigma} t_{i j} a_{i \sigma}^{\dagger} a_{j \sigma}+U \sum_{i} n_{i \uparrow} n_{i \downarrow} \\
& +\sum_{i<j} K_{i j} n_{i} n_{j}+\sum_{i<j} \frac{2}{R_{i j}},
\end{aligned}
$$

where $t_{i j}$ is the hopping integral, $U$ - the intraatomic interaction magnitude, $\epsilon_{a}$ - the atomic energy per site, and $2 / R_{i j}=2\left|\boldsymbol{R}_{j}-\boldsymbol{R}_{i}\right|^{-1}$ - ion-ion interaction corresponding to the classical Coulomb repulsion (in atomic units).

We have the total number of electrons $N_{\mathrm{e}}=\sum_{i} n_{i}$, and define the deviation from one-electron-per-atom configuration $\delta n_{i}=n_{i}-1$. We rearrange [16]:

$$
\begin{aligned}
& \sum_{i<j} K_{i j} n_{i} n_{j}=\sum_{i<j} K_{i j} \delta n_{i} \delta n_{j}+N_{\mathrm{e}} \frac{1}{N} \sum_{i<j} K_{i j} \\
& \quad+\left(N_{\mathrm{e}}-N\right) \frac{1}{N} \sum_{i<j} K_{i j} .
\end{aligned}
$$

For half band-filling $n=N_{\mathrm{e}} / N=1$ the latter part disappears, and we can write $\sum_{i<j} K_{i j} \approx \sum_{i<j} K_{i j} n_{i} n_{j}$, thus introducing the effective atomic energy per site $\epsilon_{a}^{\mathrm{eff}}=\epsilon_{a}+\frac{1}{N} \sum_{i<j}\left(K_{i j}+\frac{2}{R_{i j}}\right)$. Let us rewrite the Hamiltonian 1 in a following manner:

$$
\begin{aligned}
\mathcal{H} & =\epsilon_{a}^{\mathrm{eff}} \sum_{i} n_{i}+\sum_{i \neq j, \sigma} t_{i j} a_{i \sigma}^{\dagger} a_{j \sigma}+U \sum_{i} n_{i \uparrow} n_{i \downarrow} \\
& +\frac{1}{2} \sum_{i \neq j} K_{i j} \delta n_{i} \delta n_{j} .
\end{aligned}
$$

Since we are interested in calculating explicitly the aver- 
age value $\langle\mathcal{H}\rangle$, we note that close to the metal-insulator boundary $\left\langle\delta n_{i} \delta n_{j}\right\rangle \approx 0$, hence we disregard this term in the calculation of energy.

\subsection{Wave-function optimization}

To calculate the microscopic parameters $\epsilon_{a}, t_{i j}$, $K_{i j}, U$ of the Hamiltonian (3) we choose the basis of the orthogonalized-to-the-nearest-neighbors Wannier $w_{i}$ functions constructed from $1 s$ Slater-type orbitals (STO) $\Psi_{i}$ :

$$
w_{i}(\boldsymbol{r})=\beta \Psi_{i}(\boldsymbol{r})-\gamma \sum_{j=1}^{z} \Psi_{j}(\boldsymbol{r}),
$$

where $\beta$ and $\gamma$ (see [10] Eqs. (24) and (25)) are mixing parameters specified for the topology of the crystal, and depending explicitly on the overlap integrals of the single-particle functions. $z$ is the number of nearest neighbors.

Obtaining the microscopic parameters from the first principles requires several integrations, since

$$
\begin{aligned}
\epsilon_{a} & =\left\langle w_{i}\left|H_{1}\right| w_{i}\right\rangle, \quad t_{i j}=\left\langle w_{i}\left|H_{1}\right| w_{j}\right\rangle, \\
U & =\left\langle w_{i} w_{i}|2| \boldsymbol{r}_{1}-\left.\boldsymbol{r}_{2}\right|^{-1} \mid w_{i} w_{i}\right\rangle,
\end{aligned}
$$

$$
K_{i j}=\left\langle w_{i} w_{j}|2| \boldsymbol{r}_{1}-\left.\boldsymbol{r}_{2}\right|^{-1} \mid w_{i} w_{j}\right\rangle,
$$

where $H_{1}$ is the Hamiltonian for a single particle in the system, and $2\left|\boldsymbol{r}_{1}-\boldsymbol{r}_{2}\right|^{-1}$ interparticle interaction in atomic units. Calculating (5) with basis as given in (4) requires solving very complicated series of integrals and can be simplified by approximating STO with a series of Gaussian functions

$$
\begin{aligned}
& \Psi_{i}(\boldsymbol{r})=\sqrt{\frac{\alpha^{3}}{\pi}} \mathrm{e}^{-\alpha\left|\boldsymbol{r}-\boldsymbol{R}_{\boldsymbol{i}}\right|} \approx \\
& \alpha^{\frac{3}{2}} \sum_{a=1}^{p} B_{a}\left(\frac{2 \Gamma_{a}^{2}}{\pi}\right)^{\frac{3}{4}} \mathrm{e}^{-\alpha^{2} \Gamma_{a}^{2}\left|\boldsymbol{r}-\boldsymbol{R}_{i}\right|^{2}},
\end{aligned}
$$

where $B_{a}$ and $\Gamma_{a}$ are parameters found by minimizing energy of the single atom $\left(\mathcal{H}_{1} \stackrel{\text { a.u. }}{=}-\nabla^{2}-2\left|\boldsymbol{r}-\boldsymbol{R}_{\boldsymbol{i}}\right|^{-1}\right)$. $p$ is a number of Gaussian functions used for the approximation. $\alpha$ is the inverse function size and will remain a variational parameter, allowing us to renormalize the ground state function to find the minimal energy for given lattice

\begin{tabular}{|c|c|c|c|c|c|c|c|c|}
\hline \multicolumn{2}{|c|}{ STO-3G } & \multicolumn{2}{|c|}{ STO-5G } & \multicolumn{2}{|c|}{ STO-7G } & \multicolumn{2}{|c|}{ STO-9G } & \multirow[b]{2}{*}{$a$} \\
\hline$B_{a}$ & $\Gamma_{a}^{2}$ & $B_{a}$ & $\Gamma_{a}^{2}$ & $B_{a}$ & $\Gamma_{a}^{2}$ & $B_{a}$ & $\Gamma_{a}^{2}$ & \\
\hline 0.7079069 & 0.4037496 & 0.4862397 & 0.3428813 & 0.3347926 & 0.3073439 & 0.2333815 & 0.2832535 & 1 \\
\hline 0.3460096 & 0.897739 & 0.4687430 & 0.6489746 & 0.4947580 & 0.5341995 & 0.4735227 & 0.4656983 & 2 \\
\hline 0.0691531 & 1.9705714 & 0.1446282 & 1.2283203 & 0.2218991 & 0.9285009 & 0.2825582 & 0.7656564 & 3 \\
\hline & & 0.0307340 & 2.3248533 & 0.0674427 & 1.6138428 & 0.1065788 & 1.2588187 & 4 \\
\hline & & 0.0093803 & 4.4002717 & 0.0188009 & 2.8050467 & 0.0341750 & 2.0696289 & 5 \\
\hline & & & & 0.0038829 & 4.8754978 & 0.0099417 & 3.4026852 & 6 \\
\hline & & & & 0.0018480 & 8.4741829 & 0.0032307 & 5.5943683 & 7 \\
\hline & & & & & & 0.0006094 & 9.1977233 & 8 \\
\hline & & & & & & 0.0004466 & 15.1220138 & 9 \\
\hline
\end{tabular}
parameter $R$. For the sake of completeness we explicitly illustrate the quality of the approximation (Fig. 1) and the coefficient for different STO- $p \mathrm{G}$ basis (Table I).

\section{TABLE I}

$B_{a}$ and $\Gamma_{a}$ coefficient obtained by minimizing the single-particle energy with wave functions given by (6).

\subsection{Ground-state energy}

As stated earlier we would like to determine the inverse wave function size $\alpha$ minimizing the ground-state energy. To obtain the values for given $\alpha$ and the fixed lattice parameter $R$ we use statistically-consistent Gutzwiller approximation (SGA) [17]. We extend the Gutzwiller approximation Hamiltonian

$$
\mathcal{H}_{\mathrm{GA}}=\epsilon_{a}^{\mathrm{eff}} \sum_{i \sigma} n_{i \sigma}+\sum_{i j \sigma} t_{i j} q_{\sigma} a_{i \sigma}^{\dagger} a_{j \sigma}+N U d^{2},
$$

where the double occupancy number $d^{2}=\left\langle n_{i \uparrow} n_{i \downarrow}\right\rangle$ and $q_{\sigma}=2\left(d \sqrt{1-2 d^{2}-m}+\sqrt{d^{2}\left(1-2 d^{2}+m\right)}\right)^{2} /\left(1-m^{2}\right)$ for $n=1$, by introducing the Lagrange-multiplier constraints

$$
\mathcal{C}_{\lambda}=-\lambda_{m} \sum_{i}\left(m_{i}-m\right)-\lambda_{n} \sum_{i}\left(n_{i}-n\right),
$$

where $m_{i} \equiv n_{i \uparrow}-n_{i \downarrow}, m \equiv\left\langle m_{i}\right\rangle, n_{i} \equiv n_{i \uparrow}+n_{i \downarrow}$, and $n \equiv\left\langle n_{i}\right\rangle$.

Finally, we use the operator $\mathcal{K}=\mathcal{H}_{G A}+\mathcal{C}_{\lambda}$ as our effective Hamiltonian. Mean fields $d^{2}$ and $m$, as well as the Lagrange multipliers $\lambda_{m}$ and $\lambda_{n}$, and the chemical potential $\mu$ are all determined variationally.

Once the ground-state energy is found as a minimal value for some $\alpha_{\min }$, we get the set of values - the microscopic parameters (5) in the ground state. Below we discuss the properties of our results in comparison to those obtained earlier [12]. 


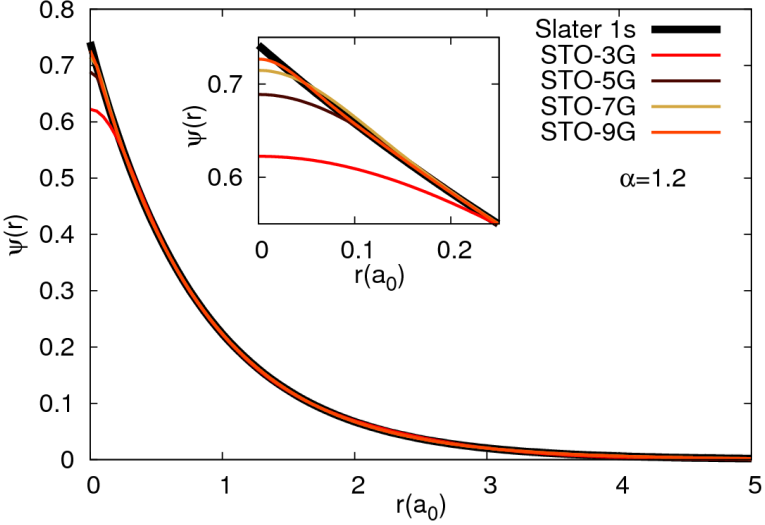

Fig. 1. Approximations of Slater $1 s$ function centered on-site with different Gaussian resolution $p$ (see (6) and Table I) for $\alpha=1.2$ with respect to distance $r$ from the ion. Inset: details for small distances. Note that the biggest contribution to the error is given by the part close to the node, hence small total error after integrating over whole space. As expected the 9 Gaussian basis (STO-9G) is far the best approximation.

\section{Gaussian basis resolution}

In our previous approach [12] we favored the Gaussian basis consisting of 3 functions. We argued that the quality of such an approximation is sufficient, and that the numerical effort to obtain results in higher Gaussian resolutions $(p>3)$ is unnecessary. The computational complexity scales

$$
\begin{aligned}
& \epsilon_{a}, t \propto p^{2}, \\
& U, K_{i j} \propto p^{4},
\end{aligned}
$$

where $p$ is the resolution. Hence the time of calculating the full set of data points is increased by a factor of 200 when replacing STO-3G to STO-9G basis.

\section{1. $S T O-3 G$ versus $S T O-9 G$}

For our $a b$ initio calculations we have selected STO-9G basis. It is much better (cf. Fig. 1) than STO-3G, while time of the calculation is still acceptable.

The dependence of the ground-state energy $E_{\mathrm{G}}$ with respect to the lattice parameter $R$ (Fig. 2) is the main outcome. Similarly to the previous case [12], there are two local minima - one associated with the metallic phase $\left(d^{2} \neq 0\right)$, and one with the Mott insulating phase $\left(d^{2}=0\right)$. The transition occurs at $R=R_{\mathrm{C}}=4.12 a_{0}$ (compared to $R_{\mathrm{C}}^{\text {old }}=4.1 a_{0}$ ), but its nature is not changed, as it still is a weakly discontinuous transition (observe the obvious discontinuity of double occupancy number, cf. inset in Fig. 2).

In Fig. 3 we plot the values of the nearest-neighbor hopping $(-t)$, on-site repulsion $U$, and the nearest-neighbor intersite repulsion $K$. Even though there are no qualitative changes in the values in comparison with [12] we present this for the sake of completeness.

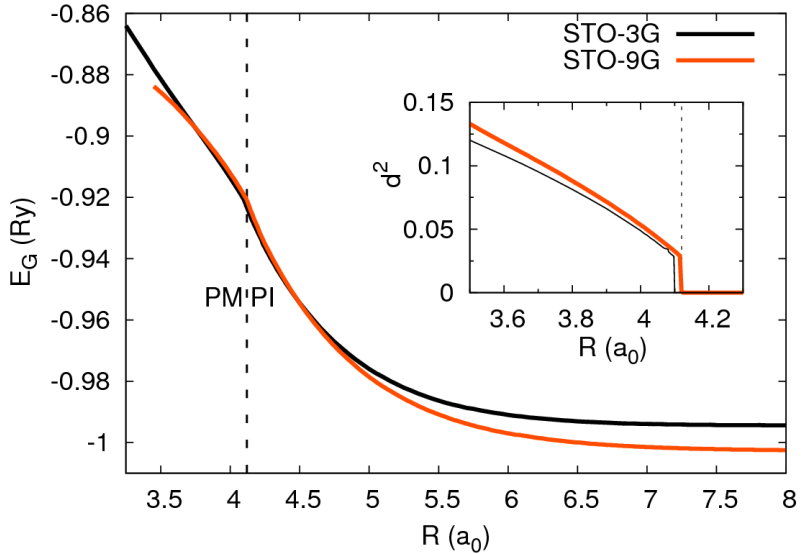

Fig. 2. Ground-state energy versus lattice parameter $R$ for different STO- $p \mathrm{G}$ basis. Note more realistic behavior in the metallic $\left(R<R_{\mathrm{C}}=4.12 a_{0}\right)$ regime with non-trivial $R$ dependence. Inset: double occupancy mean field versus lattice parameter $R$ for different STO$p \mathrm{G}$ basis. Note no qualitative changes of behavior.

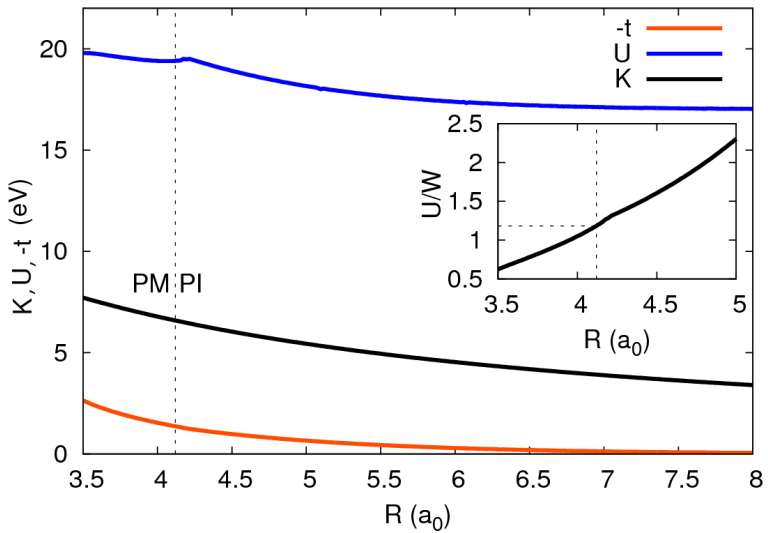

Fig. 3. The microscopic parameters $t, U$ and $K$ versus lattice parameter $R$. Inset: $U / W$ ratio with bandwidth $W=2 z|t|$ and on-site repulsion $U$.

In Ref. [12] we have shown that our transition satisfies both the Mott and the Hubbard criteria for metalinsulator transition. Below we refer to them while discussing the new results.

\subsection{The Mott and the Hubbard criteria}

The original Mott criterion $[5,6] n_{\mathrm{C}}^{1 / 3} a_{\mathrm{B}} \approx 0.2$ can be rewritten by substituting $\alpha^{-1}$ for the effective Bohr radius $a_{\mathrm{B}}$ and defining the particle density as $n_{\mathrm{C}}=R_{\mathrm{C}}^{-3}$. We get $n_{\mathrm{C}}^{1 / 3} a_{\mathrm{B}}=R_{\mathrm{C}}^{-1} \alpha^{-1} \approx 0.22$, a slightly better outcome than in [12] (as it is predicted with a better accuracy).

As shown in inset to Fig. 3, the ratio $(U / W)$ for critical lattice parameter $R_{\mathrm{C}}=4.12 a_{0}$ is equal to 1.18 in consistence with [13]. 


\subsection{Metallization pressure}

Our model represents a 3-dimensional simple-cubic crystal of the atomic hydrogen (one electron per ion, $1 \mathrm{~s}$ orbitals) undergoing the Mott-Hubbard transition. It is clear that the minimal value of energy (cf. Fig. 2) of such a crystal is reached for lattice parameter $R \rightarrow \infty$. Thus one require external pressure $p$ for its stabilization, that can be obtained classically as the force per cell $F=\left|-\nabla_{\mathrm{R}} E_{\mathrm{G}}\right|$ over the elementary cell area $A / N=R^{2}$. In Fig. 4 we plot such pressure versus lattice parameter $R$ and provide a comparison between the previously obtained (STO-3G [12]) results and the new ones.

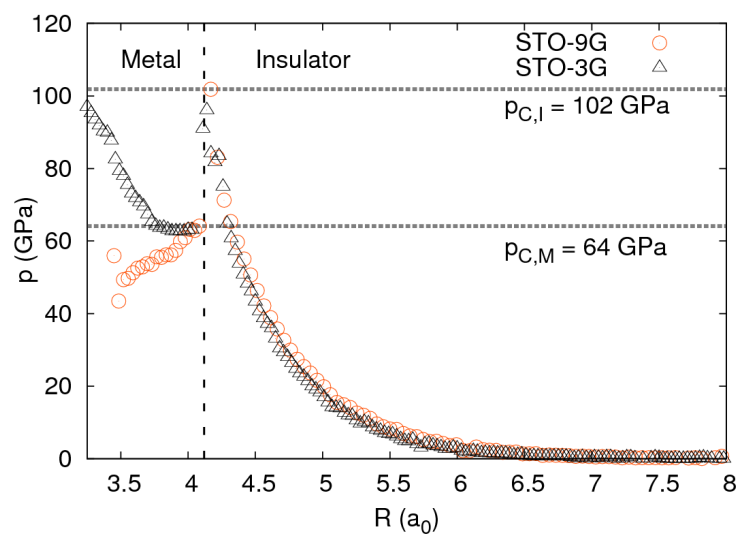

Fig. 4. Stabilizing pressure for a simple-cubic atomic solid hydrogen crystal versus lattice parameter $R$ for different STO-nG basis. Note only a slight change in obtained critical pressure $p_{\mathrm{C}}=102 \mathrm{GPa}$ for significantly larger STO-9G basis. The qualitatively different behavior of stabilizing pressure in the metallic ( $\left.R<R_{\mathrm{C}}=4.12 a_{0}\right)$ regime is caused by non-trivial behavior of energy in this regime (see Fig. 2 for details).

We have calculated the metallizing pressure $p_{\mathrm{C}}=102$ GPa assuming that our model is static - this assumption is not quite correct within the quantum-mechanical world, where there is always a non-zero energy of zeropoint oscillations. In the next section we deal with this problem by estimating the contribution of zero-point motion to the total energy.

\section{Zero-point motion energy}

We introduce (following approach similar to [18]) the uncertainties of the momentum $\delta \boldsymbol{P}$ and position $\delta \boldsymbol{R}$. The energy of a distortion per ion is

$$
\begin{aligned}
\Delta E & =\frac{\delta \boldsymbol{P}^{2}}{2 M_{H^{+}}} \\
+\frac{1}{2} & \sum_{i \in\{x, y, z\}}\left(\frac{e^{2}}{R+\delta R_{i}}+\frac{e^{2}}{R-\delta R_{i}}\right) .
\end{aligned}
$$

By applying the uncertainty relation $\delta \boldsymbol{P}^{2} \cdot \delta \boldsymbol{R}^{2} \geq 3 \hbar^{2} / 4$ and minimizing 10 with respect to $R_{i}$ 's we get a set of local extrema, from which the global minimum is

$$
\begin{aligned}
& \Delta E_{0}=3 \frac{e^{2}}{R}+\frac{\hbar(4 \sqrt{6} e M R+\sqrt{M} \sqrt{R} \hbar)}{8 M^{3 / 2} R^{5 / 2}}, \\
& \left|\delta \boldsymbol{R}_{0}\right|=\sqrt{\frac{3 R^{2}}{2 \sqrt{6} \frac{e}{\hbar} \sqrt{M} \sqrt{R}+1}},
\end{aligned}
$$

where $R$ is the lattice parameter. The first term of (11) is related to the Coulomb repulsion of ions and the second $E_{\mathrm{ZPM}} \equiv \Delta E_{0}-3 e^{2} R^{-1}$ is the zero-point oscillation energy.

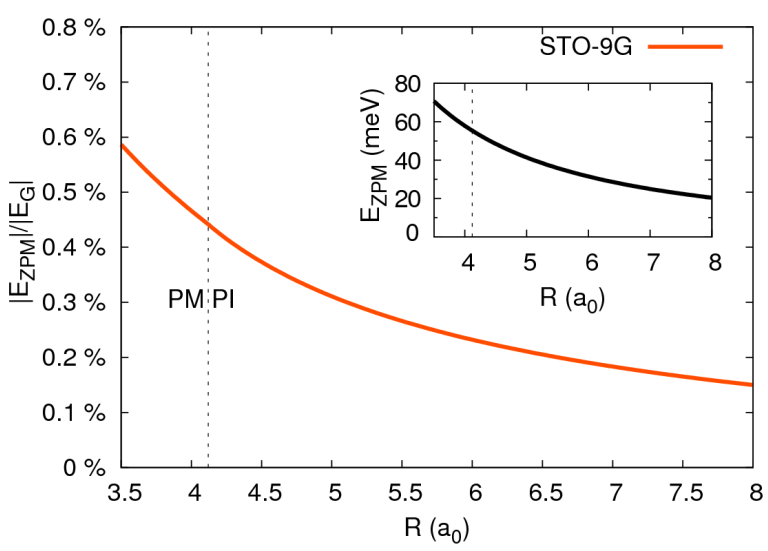

Fig. 5. The relative magnitude of estimated zero-point motion energy with respect to ground-state energy at given lattice parameter $R$. Note that result below 0.5 at the metal-insulator transition shows that the correction from ZPM to the critical pressure can be disregarded. Inset: explicit value of estimated zero-point motion energy.

In Fig. 5 we show the ratio of $\left|E_{\mathrm{ZPM}}\right|$ to the groundstate energy $\left|E_{\mathrm{G}}\right|$. Since it is slowly-changing and is about two orders of magnitude smaller than the groundstate energy, our approach of omitting it in the calculation of metallization pressure holds.

\section{Conclusions}

In this paper we established that the choice of the STO-3G basis in [12] was not influencing results qualitatively, and that the computational simplicity and total CPU time conservation are allowing us to examine also a full picture with an external magnetic field, preserving main properties of the system. Better accuracy (Sect. 2.1) increases the quality of the results (cf. Fig. 2), but does not change our understanding of the metalinsulator transition in this model.

The analysis of zero-point motion carried out in Sect. 4 reinforces our previous results and suggests that the energy of oscillations does not increase the stabilization pressure significantly. 


\section{Acknowledgments}

I would like to thank Prof. Józef Spałek for critical reading of this paper as well as Dr. Andrzej Biborski and Marcin Abram for discussions.

The work was realized as a part of the TEAM project awarded to our group by the Foundation for Polish Science (FNP) for the years 2011-2014.

\section{References}

[1] J. Hubbard, Proc. R. Soc. (London) 276, 238 (1963).

[2] M.C. Gutzwiller, Phys. Rev. Lett. 10, 159 (1963).

[3] M.C. Gutzwiller, Phys. Rev. 137, A1726 (1965).

[4] J. Kanamori, Prog. Theor. Phys. 30, 275 (1963).

[5] N.F. Mott, Proc. Phys. Soc. Sec. A 62, 416 (1949).

[6] N.F. Mott, Metal-Insulator Transitions, 2nd ed., Taylor and Francis, London 1990.

[7] F. Gebhard, The Mott Metal-Insulator Transition, Springer, Berlin 1997.
[8] M. Imada, A. Fujimori, Y. Tokura, Rev. Mod. Phys. 70, 1039 (1998).

[9] I. Bloch, Understanding Quantum Phase Transitions, Ed. L.D. Carr, CRC Press, Boca Raton 2011, Ch. 19.

[10] J. Kurzyk, W. Wójcik, J. Spałek, Eur. Phys. J. B 66, 385 (2008), Part I.

[11] J. Spałek, J. Kurzyk, R. Podsiadły, W. Wójcik, Eur. Phys. J. B 74, 63 (2010), Part II.

[12] A.P. Kądzielawa, J. Spałek, J. Kurzyk, W. Wójcik, Eur. Phys. J. B 86, 252 (2013), Part III.

[13] J. Hubbard, Proc. R. Soc. (London) 281, 401 (1964).

[14] N.W. Ashcroft, Phys. Rev. Lett. 21, 1748 (1968).

[15] E. Wigner, H.B. Huntington, J. Chem. Phys. 3, 764 (1935).

[16] A. Rycerz, Ph.D. thesis, Jagiellonian University 2003.

[17] J. Jędrak, J. Kaczmarczyk, J. Spałek, arXiv:1008.0021 2010.

[18] J. Spałek, R. Podsiadły, W. Wójcik, A. Rycerz, Phys. Rev. B 61, 15676 (2000). 